\title{
Optical fiber delay lines in microwave photonics: sensitivity to temperature and means to reduce it
}

\author{
Meng Ding, Zitong Feng, David Marpaung, Xi Zhang, Matěj Komanec, Dmytro Suslov, Daniel \\ Dousek, Stanislav Zvánovec, Eric R. Numkam Fokoua, Thomas D. Bradley, Francesco Poletti, David \\ J. Richardson, Fellow, IEEE, Fellow OSA, and Radan Slavík, Senior Member, IEEE, Fellow OSA
}

\begin{abstract}
One of the key functionalities in microwave photonics is to be able to define controllable time delays during the signal processing. Optical fibers are often used to achieve this functionality, especially when a long delay or a widely-tunable delay is needed. However, the stability of this delay in the presence of environmental changes (e.g., temperature) has not, to the best of our knowledge, been reviewed yet. Here, we firstly discuss the impact of temperature-induced variations on the signal propagation time in optical fibers and its implications in microwave photonics. We compare the impact of the thermal sensitivity of various delay lines for applications in which the signal is transported from point $A$ to point $B$, as well as for applications in which the propagation time through a fiber or the fiber dispersion is used to create a fixed or tunable delay. In the second part of the paper we show the impact of fiber thermal sensitivity on a narrow-band microwave photonics filter made of standard single mode fiber (SSMF) and a hollow core fiber (HCF), which has significantly lower thermal sensitivity of propagation time to temperature. The central frequency of the band-pass filter changes almost 16 times more in the filter made of SSMF as compared to that of $\mathrm{HCF}$, dictating very tight $\left(0.05^{\circ} \mathrm{C}\right)$ temperature stabilization for SSMF- based filters. On the basis of our thermal sensitivity analysis we conclude that HCFs are very promising for environmentally stable microwave photonics applications.
\end{abstract}

Index Terms-Microwave Photonics, Microwave filters, Optical fiber applications, Fabry-Perot

\section{INTRODUCTION}

$\mathrm{M}$ icrowave photonics uses optical devices and techniques for advanced processing and transport of radio frequency (RF) signals. Although integrated microwave photonics is compelling due to advantages such as small size and compactness, optical fibers still play a key role due to their ultralow loss (particularly relevant for long distances). One of the key microwave photonics requirements is to be able to set a

\footnotetext{
$\mathrm{M}_{\text {anuscirpt }}$ received December $\mathrm{xx}, \mathrm{xxxx}$; revised February $\mathrm{xx}, \mathrm{xxxx}$; accepted $\mathrm{xx}, \mathrm{xxxx}$. This work was supported by EPSRC project "Airguide Photonics", under grant EP/P030181/1, Czech Technical University in Prague (SGS SGS17/182/OHK3/3T/13) and MEYS INTER-COST project LTC18008 within COST 16220 EUIMWP. Meng Ding is supported by CSC scholarship. Francesco Poletti gratefully acknowledges EU ERC (grant 682724); Radan Slavík was supported by RAEng Fellowship.

Meng Ding, Zitong Feng, Eric R. Numkam Fokoua, Xi Zhang, Thomas D. Bradley, Francesco Poletti, David J. Richardson, and Radan Slavík are with the
}

a controllable delay of the signal being processed [1]. Defining a fixed delay is straightforwardly realized with minimum loss or signal distortion via signal propagation through a length of an integrated optics waveguide or an optical fiber. Some microwave photonics applications, however, require a tunable delay line, which is more challenging to implement. Quasitunable delay lines have been implemented, e.g., using a set of fiber lengths and switches with a fixed wavelength laser, Fig. 1(a) [1], while a continuously tunable delay line can be achieved, e.g., with a dispersive fiber and a tunable laser source, Fig. 1(b) [2].

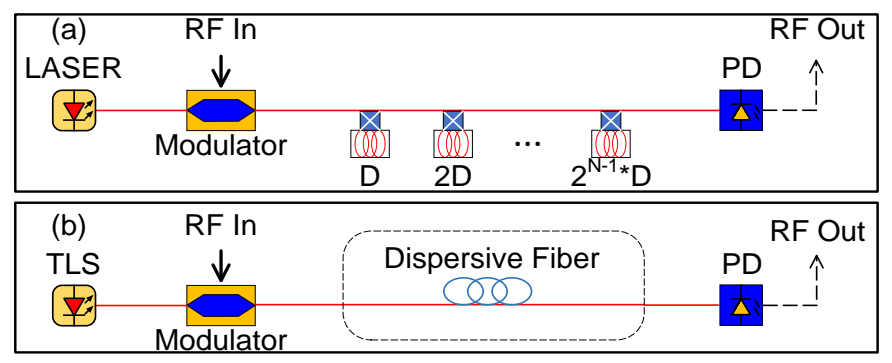

Fig. 1 Principle of tunable delay lines based on (a) switched fiber lengths with a fixed laser wavelength, and (b) a dispersive delay line with tunable laser source. D: delay; PD: photodetector; TLS, tunable laser source.

Delay lines in microwave photonics are often used to construct filters in finite input response (FIR) configurations [2], whose schematic is shown in Fig. 2 (a). By combining several (at least two) replicas of the original signal (delayed with respect to each other using delay lines) with adjusted amplitudes and phases, a filtering functionality is achieved. Indeed, infinite impulse response (IIR) configurations [2] that use output signal (after an appropriate delay) fed back into the input are also used, Fig. 2 (b). When combined with gain, such feedback structures can also be made to oscillate, forming an optoelectronic oscillator (OEO) [3]. Fig. 2(c) shows a schematic of a simple OEO. As its noise properties are directly

Optoelectronic Research Centre, University of Southampton, SO17 1BJ, UK (Zitong.Feng@soton.ac.uk; R.Slavik@soton.ac.uk).

David Marpaung is with the Nonlinear Nanophotonics group, University of Twente, the Netherlands.

Matěj Komanec, Dmytro Suslov, Daniel Dousek, and Stanislav Zvánovec are with the Faculty of Electrical Engineering, Czech Technical University in Prague, 16627 Prague, Czech Republic. 
related to the magnitude of the delay introduced in the feedback loop [3], a long delay (e.g., created in km-length fibers) is often used.

For stable operation of microwave photonics filters and other related devices such as OEOs, the delay introduced by a delay line should be stable with temperature. Even when using optical fibers for transport of the microwave signal (such as clock distribution [4], 5G networks [5], or radio astronomy [6]), changes in the RF signal phase due to temperature are of importance. However, the topic of this thermal stability has not, to the best of our knowledge, been comprehensively discussed in the literature in the field of microwave photonics yet.

In this paper, we first carry out a comprehensive comparison of fiber-based microwave photonics delay lines in terms of their thermal stability. We introduce the key effects that influence the thermal stability and quantify their contribution, allowing for comprehension of the topic as well as enabling microwave photonics engineers to design their systems taking the thermal stability into account. For example, using a dispersion compensating fiber instead of a standard optical fiber in the dispersive tunable delay line, up to 6 times lower sensitivity to temperature can be obtained.In the second part, we demonstrate our thermal sensitivity analysis on an IIR microwave photonics filter based on an all-fiber Fabry-Perot etalon made of standard fiber as well as novel hollow core optical fiber (the microwave photonics filter based on hollow core fibre (HCF) is demonstrated for the first time here). Thanks to the significantly better thermal stability of the HCF as compared to standard single mode fiber (SSMF), the developed HCF based microwave filter has a more than ten times better central frequency stability. We show this is of key importance in ultranarrow pass-band filters $(180 \mathrm{kHz}$ in our filter, measured up to a central frequency of $40 \mathrm{GHz}$ ) and how this scales with the operational frequency.
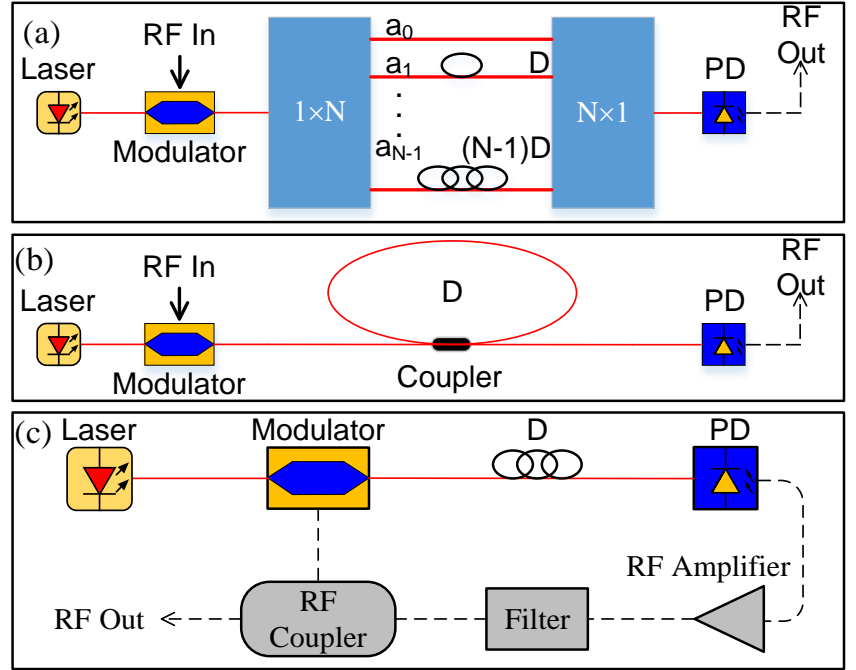

Fig. 2 Schematic of a FIR (a) and IIR (b) microwave photonics filters [2] and a simple $\mathrm{OEO}(\mathrm{c})$.

\section{FIBERS' THERMAL SENSITIVITY}

There are several key configurations (Section III) used to generate delays in microwave photonics using optical fibers. Before analyzing their thermal sensitivities, we need to know the relevant thermal sensitivity properties of the optical fibers used and in particular of their propagation time and chromatic dispersion.

\section{A. Fiber delay}

The propagation time $\tau$ of a microwave photonics signal propagating through a length $L$ of an optical fiber or a waveguide is given by:

$$
\tau_{\text {delay }}=\frac{n_{g} L}{c},
$$

where $n_{\mathrm{g}}$ in the group refractive index of the propagating medium and $c$ is the speed of light in vacuum. When temperature $T$ changes, two effects contribute to the time delay change: the fiber elongation with temperature (thermal expansion) and the change of propagation speed with temperature [7]. The change of time delay due to the temperature change per unit length is characterized by the thermal coefficient of delay (TCD). For an SSMF, which is made of fused silica, the TCD is about $40 \mathrm{ps} / \mathrm{km} / \mathrm{K}$ to which the change of propagation speed with temperature contributes about 95\% [7]. The thermal expansion contribution is very small thanks to the extremely low thermal expansion coefficient $\left(S_{\mathrm{L}}=5 \times 10^{-7} / \mathrm{K}[8]\right)$ of silica glass, contributing about 2 $\mathrm{ps} / \mathrm{km} / \mathrm{K}$ [7]. For applications where the TCD of SSMF is too high, fibers with a specialty coating were developed [9] known as phase stable optical fiber (PSOF), reducing the TCD down to $3.7 \mathrm{ps} / \mathrm{km} / \mathrm{K}$.

Another approach is to use $\mathrm{HCF}$ in which light propagates through a central hole, eliminating the contribution of thermal induced propagation speed change, reducing the TCD to 2 $\mathrm{ps} / \mathrm{km} / \mathrm{K}$, limited by the thermally-induced fiber elongation. This advantage has already been demonstrated in microwave photonics, resulting in the demonstration of an OEO with 16 times smaller sensitivity of the oscillating frequency to temperature as compared to an OEO made with SSMFs [10]. By careful design, HCFs can even be achieved with a TCD close to $0 \mathrm{ps} / \mathrm{km} / \mathrm{K}$ [11] and an OEO with $>100$ times better thermal stability than SSMF has been reported using such a design [12]. Besides lower TCD, HCFs have other advantages over SSMFs relevant for microwave photonics, e.g., low nonlinearity in combination with low chromatic dispersion [13] The key limitation to using HCF in microwave photonics applications had been up to recently transmission loss, which used to be significantly higher compared to SSMF. However, with the development of the latest generation of hollow core fibers: nested antiresonant nodeless fibers (NANFs) [14]-[17], attenuation levels of $0.28 \mathrm{~dB} / \mathrm{km}$ have recently been achieved [17] and one can expect it to become even lower in the near future. NANF is therefore expected to be an ideal candidate for delay lines designed for microwave photonic and should open new device opportunities. However, to date, the use of NANF has not been demonstrated in any microwave photonics application to the best of our knowledge.

\section{B. Chromatic dispersion}

The chromatic dispersion of an optical fiber is given by the sum of material and waveguide dispersions [18]. For weakly- 
guiding fibers like SSMFs or typical dispersion compensation fibers (DCFs), the chromatic dispersion can be approximated by the three term Sellmeier equation [18], [19]:

$$
D(\lambda)=\frac{S_{0}}{4}\left(\lambda-\frac{\lambda_{0}^{4}}{\lambda^{3}}\right)
$$

where $\lambda_{0}$ is the zero dispersion wavelength and $S_{0}$ is the dispersion slope at the zerodispersion wavelength. The chromatic dispersion of SSMF and a typical DCF at C band obtained using Eq. (2) and fiber data from [20] and [21] are both plotted in Fig. 3. The temperature sensitivity of chromatic dispersion can be obtained by differentiating Eq. (2) with respect to temperature $T$ :

$$
\frac{d D}{d T}=\frac{D}{S_{0}} \cdot \frac{d S_{0}}{d T}-\frac{S_{0} \lambda_{0}^{3}}{\lambda^{3}} \cdot \frac{d \lambda_{0}}{d T}
$$

We then used Eq. (3) to calculate the chromatic dispersion of SSMF with a temperature increase of $100 \mathrm{~K}$ by using data from [20] (fiber Type A1 in [20]) and show it in Fig. 3. As concerns $\mathrm{DCF}$, only the parameters relevant to the zero dispersion wavelength have been published so far (e.g., in [21]), which allows us to calculate the second term in Eq. (3), but not the first one. To allow us to illustrate the DCF's thermal sensitivity, we made an assumption that the first term in Eq. (3) (proportional to the change of $\mathrm{S}_{0}$ ) is negligible as compared to the second one (proportional to the change of $\lambda_{0}$ ). We consider it a reasonable assumption, as for SSMF, the first term in Eq. (3) typically contributes to only $5-9 \%$ of the overall thermal sensitivity in the C-band [21]. Although it may have a larger contribution for the DCF, it is unlikely to be a dominant term.

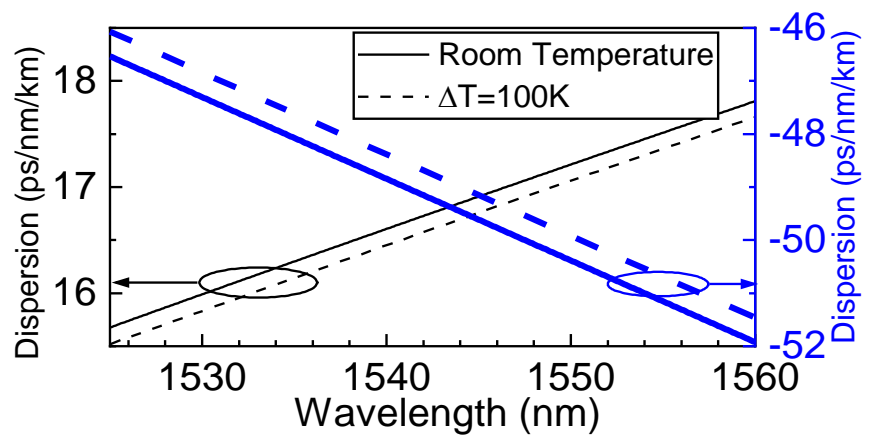

Fig. 3 Chromatic dispersion of SSMF (thin black) and DCF (thick blue, at room temperature calculated with Eq. (2) and data from [20], [21]. Chromatic disperiosn at temperature $100 \mathrm{~K}$ higher for SSMF was calculated with Eq. (3) and data from [20] (thin black dashed) and DCF calculated from the second term of Eq. (3) and data from [21] (thick blue dashed).

In Fig. 3 we see that over a limited bandwidth (e.g., C-band in Fig. 3) the dispersion curve is mainly red-shifted when the temperature is increased. Although this observation has been done with DCF characteristics that are approximate (as discussed earlier), it is in line with [21] and [22], where for all studied fiber types (including SSMF and DCF), the dispersion thermal sensitivity was considered to be:

$$
\frac{d D(\lambda)}{d T} \cong S(\lambda) \cdot \frac{d \lambda_{0}}{d T}
$$

where $D(\lambda)$ is the chromatic dispersion of interest and $S(\lambda)$ is the dispersion slope at that wavelength. As $\mathrm{d} \lambda_{0} / \mathrm{dT}$ is almost constant for any fiber type $\left(\mathrm{d} \lambda_{0} / \mathrm{dT}=(0.02-0.03) \mathrm{nm} / \mathrm{K}[20]-\right.$ $[24]), \mathrm{dD}(\lambda) / \mathrm{dT}$ depends predominantly on the dispersion slope $S(\lambda)$, as follows from Eq.(4).

For SSMF, chromatic dispersion and its thermal sensitivity are $17 \mathrm{ps} / \mathrm{nm} / \mathrm{km}$ (at $1550 \mathrm{~nm}$ ) and $-1.5 \mathrm{fs} / \mathrm{nm} / \mathrm{km} / \mathrm{K}$. For DCF, the chromatic dispersion ranges from -30 to $-300 \mathrm{ps} / \mathrm{nm} / \mathrm{km}$ [25] and $\mathrm{d} D(\lambda) / \mathrm{d} T$ is between 0.9 and $4 \mathrm{fs} / \mathrm{nm} / \mathrm{km} / \mathrm{K}$ [21] for $\lambda \sim 1550$ $\mathrm{nm}$. Photonic crystal fiber (PCF) generally provides larger dispersion, e.g., $-700 \mathrm{ps} / \mathrm{nm} / \mathrm{km}$ [26], but also has a larger dispersion slope $\mathrm{S}(\lambda)$ (as compared to DCF), making its $\mathrm{d} D / \mathrm{d} T$ relatively large, e.g., data from [26] leads to $\mathrm{d} D / \mathrm{d} T \sim 1.6$ $\mathrm{ps} / \mathrm{nm} / \mathrm{km} / \mathrm{K}$.

The key properties of optical fibers relevant for microwave photonics delay lines and their thermal behavior are

\begin{tabular}{|c|c|c|c|c|c|c|}
\hline & \multicolumn{4}{|c|}{ Silica-core fibers } & \multicolumn{2}{|c|}{ Hollow-core } \\
\hline & SSMF & PSOF & DCF & PCF & PBGF & NANF \\
\hline $\begin{array}{l}\text { TCD, } \\
\mathrm{ps} / \mathrm{km} / \mathrm{K}\end{array}$ & $\begin{array}{l}40 \\
{[7]}\end{array}$ & $\begin{array}{l}3.7 \\
{[9]}\end{array}$ & & & $\begin{array}{l}2.2 \\
{[10]}\end{array}$ & $1.7[13]$ \\
\hline $\begin{array}{l}\text { Loss, } \\
\mathrm{dB} / \mathrm{km}\end{array}$ & 0.2 & $\begin{array}{l}0.4 \\
{[27]}\end{array}$ & & & $\begin{array}{l}1.7 \\
{[28]}\end{array}$ & $\begin{array}{l}0.28 \\
{[17]}\end{array}$ \\
\hline $\begin{array}{l}\text { Disp., } \\
\text { ps/nm/km }\end{array}$ & 17 & & $\begin{array}{l}-30 \\
\text { to } \\
-300 \\
\end{array}$ & $\begin{array}{l}-700 \\
{[26]}\end{array}$ & $0-100$ & $2-6$ \\
\hline $\begin{array}{l}\text { Disp. } \\
\text { thermal } \\
\text { sensitivity, } \\
\text { fs } / \mathrm{nm} / \mathrm{km} / \mathrm{K}\end{array}$ & $\begin{array}{l}-1.5 \\
{[20]}\end{array}$ & & $\begin{array}{l}0.9 \\
\text { to } 4 \\
{[21]}\end{array}$ & $\begin{array}{l}1600 \\
{[26]}\end{array}$ & & \\
\hline
\end{tabular}
summarized in Table 1.

Table 1 Summary of discussed properties of opitcla fibers.

*SSMF: Standard single-mode fiber, PSOF: Phase-stable optical fiber, DCF: Dispersion-compensating fiber; PCF: Photonic crystal fiber, PBGF: Photonic bandgap fiber, NANF: Nested antiresonant nodeless fiber. All of these data are given in the $\mathrm{C}$ band.

\section{OPTICAL MICROWAVE PHOTONICS DELAY LINES AND THEIR THERMAL SENSITIVITY}

Here, we divide the key configurations to generate delay in microwave photonics into three main categories that we discuss in the following text: (i) Fiber delay, (ii) Delay via fiber chromatic dispersion, and (iii) Delay with chirped fiber Bragg gratings (CFBG).

In the literature, there are two main concepts used to characterize the thermal properties of optical fibers. The first one normalizes time delay changes to the fiber length as characterized by the TCD (used, e.g., [9]) and is appropriate for applications in which fiber is used to transport a signal from point A to point B (e.g., to synchronize telescopes in radio astronomy [6]). Fiber properties relevant to these applications are summarized in Tab. 1. The other uses normalization to unit delay (1 s). For delay lines that serve to delay a signal, normalization to delay is more appropriate: 


$$
S_{\tau}=\frac{1}{\tau} \frac{d \tau}{d T}
$$

To distinguish between the normalization to length and delay, we use the relative unit $(\mathrm{ppm} / \mathrm{K})$ for the normalization to delay (as opposed to the $\mathrm{ps} / \mathrm{km} / \mathrm{K}$ we use for normalization to the length). In the previous section (Section II), we have already compared delay lines in terms of TCD. Here, we will discuss comparison in terms of $S_{\tau}$.

\section{A. Fiber Delay}

The thermal sensitivity normalized to unit delay $S_{\tau}$ can be obtained from Eqs. (1) and (5) as:

$$
S_{\tau}=\frac{1}{n_{g}} \frac{d n_{g}}{d T}+\frac{1}{L} \frac{d L}{d T}=S_{n}+S_{L}
$$

The first term describes the sensitivity of fiber group index to temperature, the second the thermally-induced expansion.

The thermal sensitivities $S_{\tau}$ of SSMF delay lines can be caculated from the TCD published in [9] to be around $8 \mathrm{ppm} / \mathrm{K}$. A PSOF-based delay line was reported to have $S_{\tau}$ of $1.4 \mathrm{ppm} / \mathrm{K}$ [29]. HCF-based delay lines have been measured to have $S_{\tau}$ of $0.6 \mathrm{ppm} / \mathrm{K}$ using PBGF [10] and values as low as $-0.08 \mathrm{ppm} / \mathrm{K}$ have been achieved when operating the PBGF close to its zero thermal sensitivity wavelength [12]. NANF has been demonstrated to achieve $0.5 \mathrm{ppm} / \mathrm{K}$ [13].

\section{B. Delay via fiber Chromatic Dispersion}

Fig. 1(b) shows a simple schematic of a tunable dispersive fiber delay line, it's temperature sensitivity is essentially similar to that of a simple fiber delay line, Fig. 1 (a) analyzed in the previous section. However, the dispersive tunable delay line is often used in FIR filters in which signals generated by several lasers emitting at different wavelengths propagate through the same fiber and it is only the difference in their propagation times that is of importance. Fig. 4 shows a simple schematic of such a filter using two lasers with a wavelength separation of $\Delta \lambda$. Signals from both lasers are modulated by an RF signal and then the RF modulated signals at two wavelengths share the same fiber, where they experience different time delays due to the chromatic dispersion. The difference in the time delay experienced by the two lasers $\tau$ can be expressed as:

$$
\tau_{\text {disp }}=L D \Delta \lambda
$$

Here, $D$ indicates the fiber's chromatic dispersion. Since we are interested how the delay (tuned to the desired value) changes with temperature, we consider fixed wavelength separation here, leading to:

$$
S_{\tau}=\frac{1}{D} \frac{d D}{d T}+\frac{1}{L} \frac{d L}{d T}=S_{D}+S_{L}
$$

The first term is the normalized thermally induced change of dispersion and $\mathrm{dD} / \mathrm{dT}$ is exactly the thermal sensitivity of dispersion as discussed in section IIB. Its value for all the considered fiber types (SSMF: $-88 \mathrm{ppm} / \mathrm{K}$ at $1550 \mathrm{~nm}$; DCF: 20 to $-80 \mathrm{ppm} / \mathrm{K}$, and PCF: $-2800 \mathrm{ppm} / \mathrm{K}$ ) is significantly higher than the second term that represents thermally-induced elongation $(0.5 \mathrm{ppm} / \mathrm{K})$.

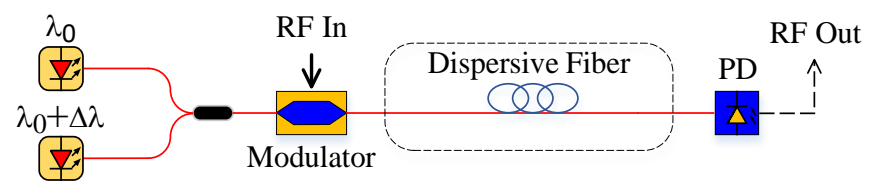

Fig. 4 Schematic of a two-tap tunable filter using a dispersive fiber structure with two lasers.

For SSMF, a tunable delay of up to $4.2 \mathrm{~ns}$ was demonstrated using $46 \mathrm{~km}$ of fiber within $5.4 \mathrm{~nm}$ range[2], while DCF with its significantly larger dispersion than SSMF (up to -300 $\mathrm{ps} / \mathrm{nm} / \mathrm{km}$ [25]), allowed a delay of up to $4 \mathrm{~ns}$ in only $1 \mathrm{~km}$ of fiber to be achieved with $40 \mathrm{~nm}$ wavelength tuning range[30]. PCFs were demonstrated to offer dispersion values of up to $700 \mathrm{ps} / \mathrm{nm} / \mathrm{km}$, enabling $60 \mathrm{ps}$ tuning using just $10 \mathrm{~m}$ of fiber [31].

The length of the fiber is limited by its loss and there are other practical considerations such as cost, volume and weight. We add to this list also thermal sensitivity, which, as we mentioned earlier, is lowest with DCF, followed by SSMF, with the worst performance obtained with PCF.

\section{Delay via Chirped Fiber Bragg Gratings}

Dispersive fiber from the previous sub-section can be substituted with a CFBG, which can have a large chromatic dispersion when operated in reflection, [32], [33]. The time delay difference between two lasers separated by $\Delta \lambda$ approximates as:

$$
\tau_{\text {chirp }} \approx\left(\frac{L}{c} \cdot \frac{1}{\Delta \Lambda_{\text {chirp }}}+\frac{L D \Delta \lambda}{n_{\text {eff }} \Delta \Lambda_{\text {chirp }}}\right) \Delta \lambda=D_{\text {chirp }} \cdot\left(1+\frac{c D \Delta \lambda}{n_{\text {eff }}}\right) \cdot \Delta \lambda(9)
$$

where $\Delta \Lambda_{\text {chirp }}$ and $L$ are the period change and the length of the CFBG respectively, and $D_{\text {chirp }}=L /\left(\mathrm{c} \cdot \Delta \Lambda_{\text {chirp }}\right)$ is the CFBG chromatic dispersion that does not take into account the fiber chromatic dispersion. $n_{\text {eff }}$ is the average effective refractive index of a CFBG. Since $L$ and $\Delta \Lambda_{\text {chirp }}$ change at the same rate with temperature (for consistency with the previous analysis, we consider a uniform temperature change along the CFBG), $D_{\text {chirp }}$ does not change with temperature. Consequently, the only parameters that change with temperature are fiber chromatic dispersion and refractive index:

$$
\frac{d \tau_{\text {chirp }}}{d T}=\frac{c D_{\text {chirp }} D(\Delta \lambda)^{2}}{n_{\text {eff }}}\left(\frac{1}{D} \frac{d D}{d T}-\frac{1}{n_{\text {eff }}} \frac{d n_{\text {eff }}}{d T}\right) .
$$

Considering a CFBG with a $D_{\text {chirp }}=250 \mathrm{ps} / \mathrm{nm}$ inscribed in SSMF as an example and $\Delta \lambda=20 \mathrm{~nm}, \mathrm{~d} \tau_{\text {chirp }} / \mathrm{d} T=-4 \times 10^{-5} \mathrm{ps} / \mathrm{K}$, equating to a thermal sensitivity $S_{\tau}$ of $0.008 \mathrm{ppm} / \mathrm{K}$, which is almost negligible.

However, real CFBGs exhibits ripples in the group delay [34], [35] due to fabrication imperfections. These ripples are 
essentially time delay deviations from the ideal CFBG response. Consequently, the time delay changes significantly with temperature. The amplitude of the group delay ripples is typically independent of the CFBG parameters and is around 10 ps [34] (but this can be reduced to 1 ps by careful apodization [36]). As temperature changes, the delay of both signals (separated by $\Delta \lambda$ ) can change by up to \pm 10 ps for a typical CFBG and \pm 1 ps for the lowest-reported ripple CFBG, generating up to $20 \mathrm{ps}$ and 2 ps peak-to-peak delay variations in $\tau_{\text {chirp }}$ respectively. As this variation does not change linearly with temperature (depends on the ripple period, grating chirp, etc.) we cannot define its sensitivity $S_{\tau}$ and can only give the peak-to-peak variations.

\section{Summary}

We have discussed various microwave photonics fiber delay lines. Table 2 summarizes the results. Although we do not cover all the microwave photonics delay line implementations, their thermal sensitivities can generally be calculated from the parameters and configurations described above.

For delay generated by signal propagation in an optical fiber, $\mathrm{HCFs}$ are the least sensitive to temperature variations. For delays generated via chromatic dispersion, a DCF based delay line is several times less thermally sensitive than a SSMF based delay line. PCF is by far the most temperature sensitive. When using a CFBG, particular attention should be paid to the group delay ripples, which determine the delay variations with temperature and even for extremely low levels of group delay ripple (1 ps) can produce a significant sensitivity to temperature. The key parameters of the delay lines just discussed in terms of $S_{\tau}$ are summarized in Table 2.

Table 2 Optical delay-lines summary

\begin{tabular}{|c|c|c|c|c|c|}
\hline & \multicolumn{2}{|c|}{ Name } & \multicolumn{2}{|c|}{ Maximum time delay* } & \multirow{2}{*}{$\begin{array}{l}S_{\tau}(\mathrm{ppm} / \mathrm{K}) \\
\sim 8\end{array}$} \\
\hline \multirow{4}{*}{ Fiber delay lines } & \multirow{2}{*}{ Solid-core fiber } & SSMF & $100 \mu \mathrm{s}$ & $L=56 \mathrm{~km}[1]$ & \\
\hline & & PSOF & $1 \mu \mathrm{s}$ & $L=0.5 \mathrm{~km} \mathrm{[32]}$ & $1 \sim 2.2$ \\
\hline & \multirow{2}{*}{ HCFs } & PBGF & $\begin{array}{l}33 \mu \mathrm{s} \\
3 \mu \mathrm{s}\end{array}$ & $\begin{array}{l}L=11 \mathrm{~km}[38] \\
L=1 \mathrm{~km}[12]\end{array}$ & $\begin{array}{l}0.6 \\
0\end{array}$ \\
\hline & & NANF & $1 \mu \mathrm{s}$ & $L=1.7 \mathrm{~km}[17]$ & 0.5 \\
\hline \multirow{4}{*}{$\begin{array}{l}\text { Dispersive fiber delay } \\
\text { lines }\end{array}$} & \multicolumn{2}{|c|}{ SSMF } & $10 \mathrm{~ns}$ & $D=17 \mathrm{ps} / \mathrm{nm} / \mathrm{km}$ & -88 \\
\hline & \multicolumn{2}{|c|}{ DCF } & $1 \mathrm{~ns}$ & $D=-300 \mathrm{ps} / \mathrm{nm} / \mathrm{km} \mathrm{[25]}$ & $-18 \sim-80$ \\
\hline & \multicolumn{2}{|c|}{ PCF } & $10 \mathrm{ps}$ & $D=-700 \mathrm{ps} / \mathrm{nm} / \mathrm{km}[31]$ & -2800 \\
\hline & \multicolumn{2}{|c|}{ CFBG } & $1 \mathrm{~ns}$ & $D_{\text {chirp }}=2500 \mathrm{ps} / \mathrm{nm}[39]$ & $\begin{array}{l}\text { Delay variations of } \\
\pm 10 \mathrm{ps}\end{array}$ \\
\hline
\end{tabular}

* Maximum delay limited by maximum fiber length $L$ or chromatic dispersion ( $D$ or $D_{\text {chirp }}$ ).

\section{NOVEL HOLLOW CORE FIBER MICROWAVE FILTER AND ITS TEMPERATURE SENSITIVITY}

In this section, we show an example of a microwave photonics filter in which the thermal stability of the fiber used is important. We fabricate the same filter using SSMF as well as HCF and compare their performance, showing that the SSMF-based filter embodiment would only operate reliably with high-precision temperature stabilization, increasing its cost, size, and power consumption.

\section{A. Set-up}

The core of our filter consists of an alignment-free all-fiber Fabry-Perot (FP) etalon made of 5-m of NANF, which has been reported in detail in [13]) or a 3.6-m long FC/PC-connectorized SSMF with mirrors directly-deposited on the FC/PC connector end-facets [40]. Due to the refractive index $(n)$ difference $(\sim 1$ for HCF and $\sim 1.45$ for SSMF), the optical length $(n L)$ of the two FP etalons is nominally identical, allowing for a fair comparison. The cross-sectional electron microscope (SEM) image of the HCF we used (NANF design, with similar parameters to that used in [16]), is shown in Fig. 5.

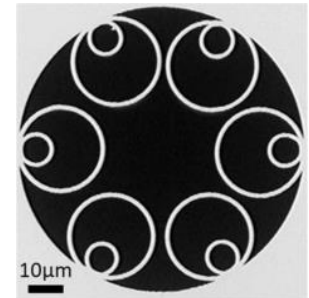

Fig. 5 The cross-sectional electron microscope (SEM) image of the NANF used in our FP etalon.

The schematic of our microwave photonics filter (we refer to it later simply as a 'filter') is shown in Fig. 6. A signal from a continuous-wave fiber laser (Rock from NP Photonics) with an output power of $16 \mathrm{dBm}$ passes through a 40-GHz bandwidth Mach-Zehnder modulator (MZM), which generates two sidebands at the frequency of the RF input signal. After passing through a polarization controller (PC), the signal is injected into the FP. The laser central frequency is set (we explain later how we set this) to be resonant with the FP, i.e., to have minimum transmission loss. Both sidebands experience the same transmission loss - i.e., both are transmitted (when the RF signal frequency is a multiple of the FP spectral period) or both are equally attenuated. Thus, the FP output signal is amplitudemodulated with a modulation depth dependent on the RF signal frequency. The output signal from the FP is photodetected and 
then divided into two parts. One of them is the output RF signal and is received by a vector network analyzer (VNA) to measure the amplitude and phase response of the FP. The other one is used for the laser wavelength locking to a transmission peak of the FP etalon via a feedback loop consisting of a lock-in amplifier, proportional-integral (PI) controller, and a piezostretcher that controls the wavelength of the Rock fiber laser. Fig. 7 shows the transmission signal and error signal before and after turning on the locking loop.

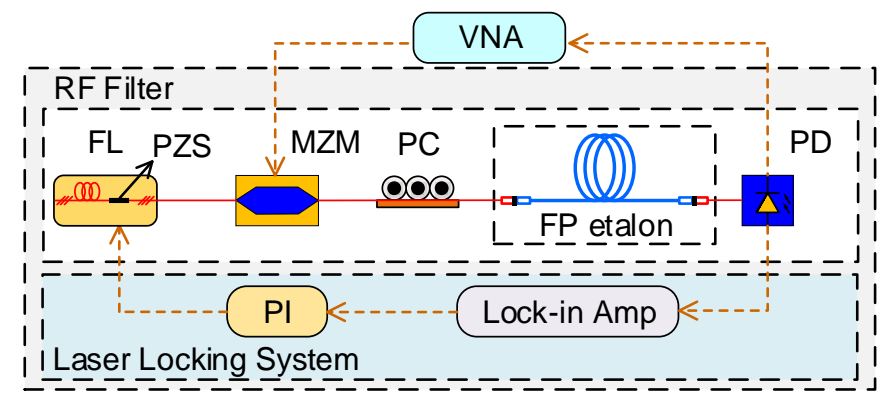

Fig. 6 Experimental set-up.FL: fiber laser; PZS: piezo-stretcher; MZM: MachZehnder modulator; PC: Polarization controller; PD: photodiode; VNA: Vector network analyzer; PI: proportional-integral controller.

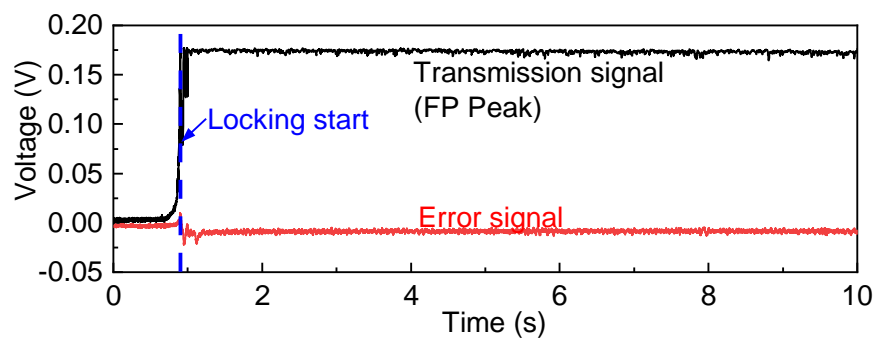

Fig. 7 Transmission signal and error signal before and after the laser wavelength has been locked to a transmission peak of the FP etalon.

\section{B. Filter transmission characteristics}

For the sake of space, we show here only the filtering characteristics for the HCF-based filter, as the filter characteristics for SSMF-based filter were very similar.

The filter has a periodic transfer function with period of 28.1 MHz (given by the FP length of $5 \mathrm{~m}$ ), shown in Fig. 8 at RF frequencies close to $10 \mathrm{GHz}, 20 \mathrm{GHz}$ and $40 \mathrm{GHz}$.

Details of the amplitude $\mathrm{S} 21$ response together with the phase response are shown in Fig. 9. The $3 \mathrm{~dB}$ passband width is $183 \mathrm{kHz}$ and the phase response has the expected $\pi$ jump at the transmission peak.

\section{Filter temperature sensitivity}

We placed the two FP etalons (based on HCF and SSMF, respectively) into the same thermal chamber. Firstly, we stabilized the temperature and measured the filters' characteristics at frequencies of $10 \mathrm{GHz}, 20 \mathrm{GHz}$ and $40 \mathrm{GHz}$. Subsequently, we increased the temperature by $1{ }^{\circ} \mathrm{C}$ and repeated the measurement, as shown in Fig. 10. The filter transmission peaks shifted in frequency. This shift was about 16 times larger for the SSMF-based filter than for HCF-based filter. As expected, the shift also depends linearly on the central frequency: i.e., the shift at $40 \mathrm{GHz}$ is 4 times larger than at 10 $\mathrm{GHz})$.
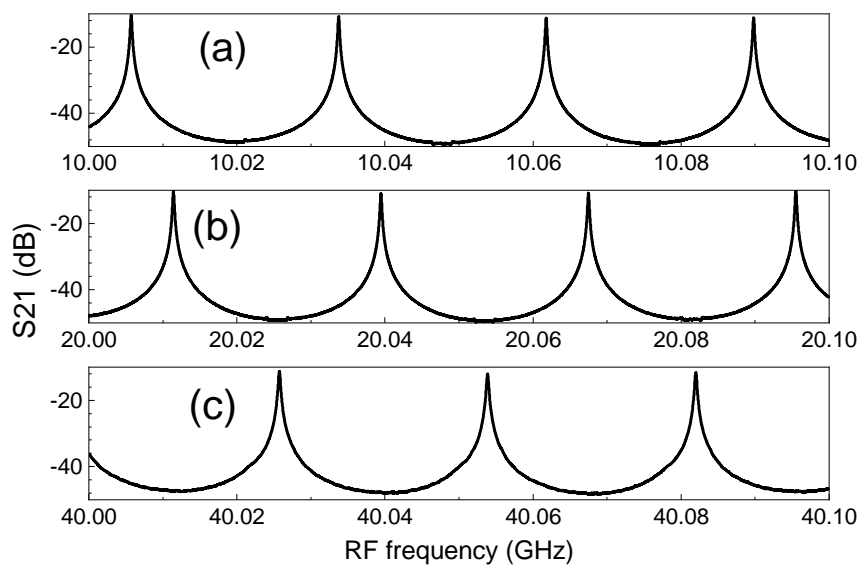

Fig. 8 Amplitude transfer characteristics of the HCF-based filter at $10 \mathrm{GHz}$ (a), $20 \mathrm{GHz}$ (b), and $40 \mathrm{GHz}$ (c) over spans of $100 \mathrm{MHz}$.

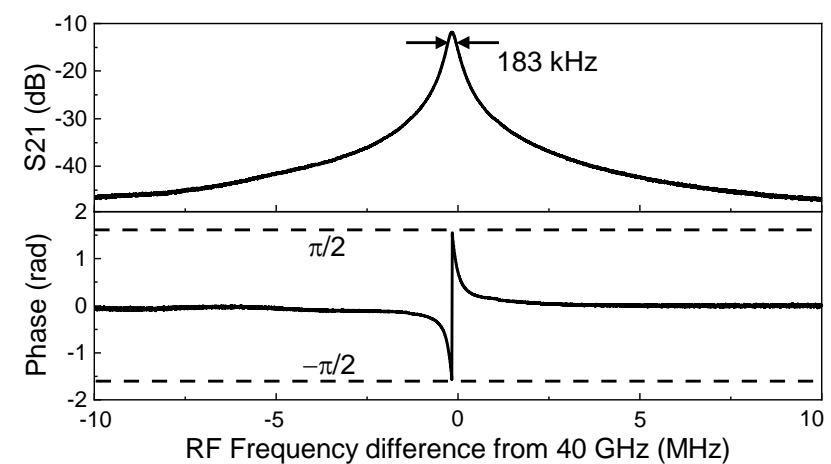

Fig. 9 Detailed amplitude and phase responses of the hollow core fiber based filter around $40 \mathrm{GHz}$ RF frequency.

To extract temperature sensitivity data with good accuracy and to confirm the shift in filter transmission characteristics is linear with temperature, we kept increasing the temperature in $1 \mathrm{~K}$ steps and measured the position of the transmission peaks. The filters transmission peak shifts (at $40 \mathrm{GHz}$, where the change is the largest) are shown in Fig. 11. For SSMF-based filter, the transmission characteristics shift at a rate of 334 $\mathrm{kHz} / \mathrm{K}$ while for the HCF-based filter the rate is only $21 \mathrm{kHz} / \mathrm{K}$ - a value almost 16 times lower as would be expected.

We also measured the filters characteristics when subject to our airconditioned lab environment (temperature variations of about $1 \mathrm{~K}$ due to the air-conditioning turning on and off). Within about an hour, the frequency variations for the SSMF based filter were up to $480 \mathrm{kHz}$ (at $40 \mathrm{GHz}$ ), while for the HCF-based filter, they were less than $30 \mathrm{kHz}$, see Fig. 12. 


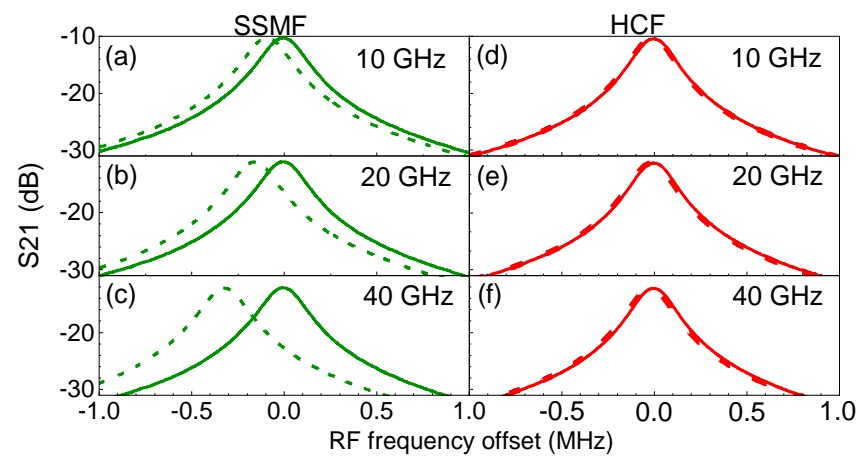

Fig. 10 Transmission characteristics measured at $25{ }^{\circ} \mathrm{C}$ (solid) and $26{ }^{\circ} \mathrm{C}$ (dashed) for SSMF-based filter (a-c, red) and HCF-based filter (d-f, green) measured at $10 \mathrm{GHz}(\mathrm{a}, \mathrm{d}), 20 \mathrm{GHz}(\mathrm{b}, \mathrm{e})$, and $40 \mathrm{GHz}(\mathrm{c}, \mathrm{f})$.

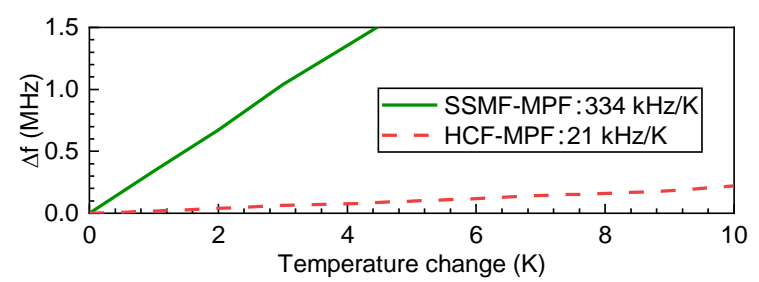

Fig. 11 Filter characteristics frequency shift at $40 \mathrm{GHz}$ over $10 \mathrm{~K}$ temperature change for HCF-based filter (dashed, red) and SSMF-based filter (solid, green). FS: Frequency Shift of RF signal

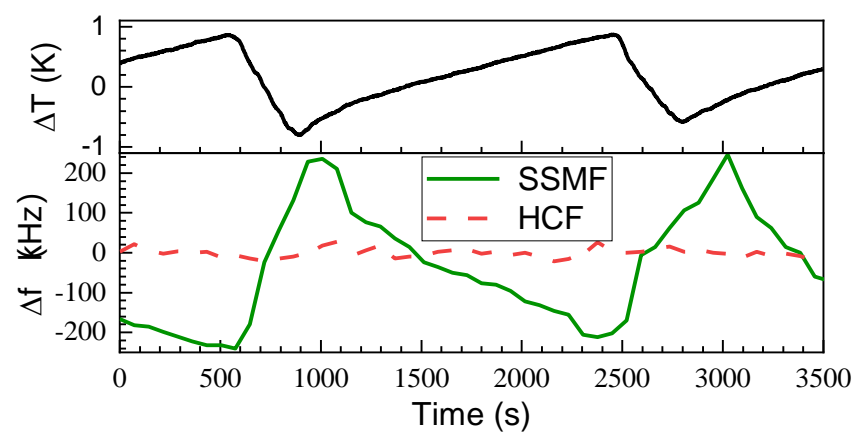

Fig. 12 Temperature variations $\Delta \mathrm{T}$ in the laboratory and measured transmission peak frequency variations $\Delta \mathrm{f}$ for HCF (solid, green) and SSMF based (dashed, red) filters when subject to laboratory environment over 1 hour.

\section{Discussion}

The results presented in Fig. 12 show that the SSMF-based bandpass filter operating at $40 \mathrm{GHz}$ changes its central frequency (by up to $480 \mathrm{kHz}$ ) by more than is its bandwidth (3-dB bandwidth of 183 $\mathrm{kHz}$ ), even in a temperature-controlled laboratory environment. On the other hand, an HCF-based filter allows for accurate operation without any further environmental stabilization.

To ensure that the central filter frequency does not change by more than $10 \%$ of its 3-dB bandwidth, the SSMF based filter (temperature shift of $334 \mathrm{kHz} / \mathrm{K}$, Fig. 11) requires temperature stabilization better than $0.05^{\circ} \mathrm{C}$, which is rather impractical. On the other hand, the HCF based filter $(21 \mathrm{kHz} / \mathrm{K}$, Fig. 11) requires temperature stabilization of just $0.9{ }^{\circ} \mathrm{C}$, easily achievable with simple temperature control. These requirements could be relaxed if the filter is operated at a lower frequency (e.g., at $10 \mathrm{GHz}$, four times larger temperature variations are acceptable), or with a larger bandwidth. However, if a lower bandwidth is targeted (e.g., in our recent work, we presented a FP fiber etalon with 10 times narrower transmission peaks [41] than presented here), the temperature stabilization needs to be improved, rendering a filter made of SSMF unstable and needing impractical mK-level temperature stabilization.

From the temperature-induced shifts given earlier $(334 \mathrm{kHz} / \mathrm{K}$ and $21 \mathrm{kHz} / \mathrm{K}$, Fig. 11), the thermal sensitivity of the used fibers (NANF hollow core fiber and SSMF) can be calculated by:

$$
S_{\tau}=-\frac{d f_{\text {peak }} / d T}{f_{\text {peak }}}
$$

Here, $f_{\text {peak }}$ indicates the measured central frequency of the filter. From our data and Eq. (11) we calculated a thermal sensitivity of 8.4 $\mathrm{ppm} / \mathrm{K}$ (SSMF) and $0.5 \mathrm{ppm} / \mathrm{K}$ (NANF), which agrees well with the previously-published data summarized in Table 2.

\section{CONCLUSIONS}

In this paper, we reviewed different configurations of optical fiber-based delay lines for microwave photonics in terms of their thermal sensitivity. The thermal sensitivity of point-topoint delay lines is best characterized by the Thermal Coefficient of Delay (TCD), which is normalized to the delay line length. Key parameters of delay lines using different optical fibers are summarized in Tab. 1, suggesting hollow core fiber (HCF) performs the best, having a TCD of as little as 2 $\mathrm{ps} / \mathrm{km} / \mathrm{K}$.

For comparison of delay lines used primarily to create a delay, we normalize their thermal sensitivity to unit delay. There are two main configurations of such fiber-based photonic delay lines: based on propagation through a length of a fiber or propagation through a dispersive fiber or a chirped fiber Bragg grating (CFBG). The key results are summarized in Tab. 2. For simple propagation through a length of a fiber, HCF performs the best (thermal sensitivity of $0.5 \mathrm{ppm} / \mathrm{K}$ ), For the dispersive delay line, devices based on dispersion compensating fiber (DCF) have the smallest thermal sensitivity (-18 -80 ppm/K). The thermal stability of a CBGF is limited by its group delay ripple, which even when strongly reduced by tight CFBG apodization is typically still at the 1-ps level.

To demonstrate the importance of the fiber thermal sensitivity in microwave photonics, we built two narrow-band IIR filters based on all-fiber Fabry-Perot etalons. The first one was made of a standard single mode fiber (SSMF), while the other one was made of low-thermally-sensitive HCF. Compared to SSMF-based filters, the HCF-based filter changes its central frequency almost 16 times less with temperature. As a result, the HCF-based filter is stable under laboratory conditions, and in real world applications would require only simple temperature control, while a SSMF-based filter would require impractically accurate/tight temperature control levels of $0.05{ }^{\circ} \mathrm{C}$.

The experiments we have presented represent the first demonstration of the latest generation of $\mathrm{HCF}$ (which was recently demonstrated to enable a loss as low as $0.28 \mathrm{~dB} / \mathrm{km}$ ) in microwave photonics. Besides the low thermal sensitivity and a loss approaching that of SSMF, HCFs also exhibit a high nonlinear threshold, making them a very promising technology for the realization of delay lines in microwave photonics. 


\section{ACKNOWLEDGMENT}

The data in this paper is accessible through the University of Southampton research repository (DOI: 10.5258/SOTON/D1708).

\section{REFERENCES}

[1] J. F. Diehl, J. M. Singley, C. E. Sunderman, and V. J. Urick, "Microwave photonic delay line signal processing," Appl. Opt., vol. 54, no. 31, p. F35, 2015.

[2] J. Capmany, B. Ortega, and D. Pastor, "A tutorial on microwave photonic filters," J. Light. Technol., vol. 24, no. 1, pp. 201-229, 2006.

[3] X. S. Yao and L. Maleki, "Optoelectronic microwave oscillator," J. Opt. Soc. Am. B, vol. 13, no. 8, p. 1725, Aug. 1996.

[4] S. Wang et al., "2 GHz clock quantum key distribution over $260 \mathrm{~km}$ of standard telecom fiber," Opt. Lett., vol. 37, no. 6, p. 1008, Mar. 2012.

[5] L. Gavrilovska, V. Rakovic, and V. Atanasovski, "Visions Towards 5G: Technical Requirements and Potential Enablers," Wirel. Pers. Commun., vol. 87, no. 3, pp. 731-757, 2016.

[6] M. Burla et al., "System integration and radiation pattern measurements of a phased array antenna employing an integrated photonic beamformer for radio astronomy applications," in Applied Optics, 2012, vol. 51, no. 7, pp. $789-802$.

[7] A. H. Hartog, A. J. Conduit, and D. N. Payne, "Variation of pulse delay with stress and temperature in jacketed and unjacketed optical fibres," Opt. Quantum Electron., vol. 11, no. 3, pp. 265-273, 1979.

[8] N. Lagakos, J. A. Bucaro, and J. Jarzynski, "Temperature-induced optical phase shifts in fibers," Appl. Opt., vol. 20, no. 13, p. 2305, 1981.

[9] M. Bousonville et al., "New phase stable optical fiber," Beam Instrum. Work., pp. 101-103, 2012.

[10] U. S. Mutugala et al., "Optoelectronic oscillator incorporating hollowcore photonic bandgap fiber," Opt. Lett., vol. 42, no. 13, p. 2647, 2017.

[11] E. N. Fokoua, M. N. Petrovich, T. Bradley, F. Poletti, D. J. Richardson, and R. Slavík, "How to make the propagation time through an optical fiber fully insensitive to temperature variations," Optica, vol. 4, no. 6, p. 659, Jun. 2017.

[12] U. S. Mutugala et al., "Hollow-core fibres for temperature-insensitive fibre optics and its demonstration in an Optoelectronic oscillator," Sci. Rep., vol. 8, no. 1, pp. 1-6, 2018.

[13] M. Ding et al., "Long-Length and Thermally Stable High-Finesse FabryPerot Interferometers Made of Hollow Core Optical Fiber," J. Light. Technol., vol. 38, no. 8, pp. 2423-2427, 2020.

[14] F. Poletti, "Nested antiresonant nodeless hollow core fiber," Opt. Express, vol. 22, no. 20, p. 23807,2014

[15] T. D. Bradley et al., "Record Low-Loss $1.3 \mathrm{~dB} / \mathrm{km}$ Data Transmitting Antiresonant Hollow Core Fibre," in 2018 European Conference on Optical Communication (ECOC), 2018, no. 1, pp. 1-3.

[16] T. D. Bradley et al., "Antiresonant Hollow Core Fibre with $0.65 \mathrm{~dB} / \mathrm{km}$ Attenuation in the C and L Telecommunication Bands," in 2019 European Conference on Optical Communication (ECOC), 2019, p. PDP3.1.

[17] G. T. Jasion et al., "Hollow Core NANF with $0.28 \mathrm{~dB} / \mathrm{km}$ Attenuation in the C and L Bands," 2020 Opt. Fiber Commun. Conf. Exhib. OFC 2020 Proc., vol. 1, no. c, pp. 4-6, 2020.

[18] P. S. Andre, A. N. Pinto, and J. L. Pinto, "Effect of temperature on the single mode fibers chromatic dispersion," in Proceedings of the 2003 SBMO/IEEE MTT-S International Microwave and Optoelectronics Conference - IMOC 2003. (Cat. No.03TH8678), 2004, vol. 3, no. July, pp. 231-234.

[19] P. S. André and A. N. Pinto, "Chromatic dispersion fluctuations in optical fibers due to temperature and its effects in high-speed optical communication systems," Opt. Commun., vol. 246, no. 4-6, pp. 303-311, 2005.

[20] M. J. Hamp, J. Wright, M. Hubbard, and B. Brimacombe, "Investigation into the temperature dependence of chromatic dispersion in optical fiber," IEEE Photonics Technol. Lett., vol. 14, no. 11, pp. 1524-1526, 2002.

[21] T. Kato, Y. Koyano, and M. Nishimura, "Temperature dependence of chromatic dispersion in various types of optical fiber," Opt. Lett., vol. 25, no. 16, p. 1156, Aug. 2000.

[22] J. Rathje and M. Andersen, "Temperature induced change in the dispersion spectrum of dispersion compensating fibers," in OFC 2003 Optical Fiber Communications Conference, 2003., 2003, vol. 2, pp. 712713 vol.2.
[23] W. H. Hatton and M. Nishimura, "Temperature Dependence of Chromatic Dispersion in Single Mode Fibers," J. Light. Technol., vol. 4, no. 10, pp. 1552-1555, 1986.

[24] K. S. Kim and M. E. Lines, "Temperature dependence of chromatic dispersion in dispersion-shifted fibers: Experiment and analysis," J. Appl. Phys., vol. 73, no. 5, pp. 2069-2074, Mar. 1993.

[25] L. Grüner-Nielsen et al., "Dispersion-compensating fibers," Journal of Lightwave Technology, vol. 23, no. 11. pp. 3566-3579, Nov-2005.

[26] Y. Jiang, X. Chen, B. Howley, M. Y. Chen, and R. T. Chen, "Effects of temperature fluctuation on highly dispersive photonic crystal fibers," Appl. Phys. Lett., vol. 88, no. 1, 2006.

[27] Linden Photonics, Inc. "STFOC Optical Cable Non-kink Crush Proof," datasheet, $2020 . \quad$ [Online]. Available: http://www.lindenphotonics.com/documents/Linden - STFOC.pdf.

[28] P. J. Roberts et al., "Ultimate low loss of hollow-core photonic crystal fibres," Opt. Express, vol. 13, no. 1, p. 236, 2005.

[29] J. J. Liu, X. P. Ma, G. X. Pei, N. Gan, and J. Sen Yang, "Phase-stabilized RF transmission system based on LLRF controller and optical delay line," Nucl. Sci. Tech., vol. 30, no. 12, pp. 1-8, 2019.

[30] X. Zhu, F. Chen, H. Peng, and Z. Chen, "Novel programmable microwave photonic filter with arbitrary filtering shape and linear phase," Opt. Express, vol. 25, no. 8, p. 9232, 2017.

[31] Y. Jiang et al., "Dispersion-enhanced photonic crystal fiber array for a true time-delay structured X-band phased array antenna," IEEE Photonics Technol. Lett., vol. 17, no. 1, pp. 187-189, Jan. 2005.

[32] B. Ortega, J. L. Cruz, J. Capmany, M. V. Andrés, and D. Pastor, "Variable delay line for phased-array antenna based on a chirped fiber grating," IEEE Trans. Microw. Theory Tech., vol. 48, no. 8, pp. 1352-1360, 2000.

[33] Y. Liu, J. Yang, and J. Yao, "Continuous true-time-delay beamforming for phased array antenna using a tunable chirped fiber grating delay line," IEEE Photonics Technol. Lett., vol. 14, no. 8, pp. 1172-1174, 2002.

[34] B. Zhou, X. Zheng, X. Yu, H. Zhang, Y. Guo, and B. Zhou, "Impact of group delay ripples of chirped fiber grating on optical beamforming networks," Opt. Express, vol. 16, no. 4, p. 2398, 2008.

[35] M. Sumetsky, B. Eggleton, and C. de Sterke, "Theory of group delay ripple generated by chirped fiber gratings," Opt. Express, vol. 10, no. 7, p. 332, 2002.

[36] N. K. Srivastava, R. Parihar, and S. K. Raghuwanshi, "Efficient Photonic Beamforming System Incorporating a Unique Featured Tunable Chirped Fiber Bragg Grating for Application Extended to the Ku-Band," IEEE Trans. Microw. Theory Tech., vol. 68, no. 5, pp. 1851-1857, 2020.

[37] D. Kwon and J. Kim, "All-fiber interferometer-based repetition-rate stabilization of mode-locked lasers to $10^{\wedge}-14$-level frequency instability and 1-fs-level jitter over 1 s," Opt. Lett., vol. 42, no. 24, p. 5186, Dec. 2017.

[38] Y. Chen et al., "Multi-kilometer Long, Longitudinally Uniform Hollow Core Photonic Bandgap Fibers for Broadband Low Latency Data Transmission," J. Light. Technol., vol. 34, no. 1, pp. 104-113, Jan. 2016.

[39] J. Zhang and J. Yao, "Broadband microwave signal processing based on photonic dispersive delay lines," IEEE Trans. Microw. Theory Tech., vol. 65, no. 5, pp. 1891-1903, 2017.

[40] M. Ding, D. J. Richardson, and R. Slavik, "Long length fibre fabry-perot interferometers and their applications in fibre characterization and temperature sensing," in 2019 Conference on Lasers and Electro-Optics Europe and European Quantum Electronics Conference, CLEO/EuropeEQEC 2019, 2019.

[41] M. Ding, E. R. N. Fokoua, T. D. Bradley, F. Poletti, D. J. Richardson, and R. Slavik, "Hollow core fiber Fabry-Perot interferometers with finesse over 3000," in 2020 Conference on Lasers and Electro-Optics (CLEO), 2020, p. 2F2P. 